\title{
The Influence of Radiation on Ice Crystal Spectrum in the Upper Troposphere
}

\author{
Xiping Zeng* \\ Goddard Earth Sciences and Technology Center, University of Maryland, Baltimore \\ County, and Laboratory for Atmospheres, NASA Goddard Space Flight Center, \\ Greenbelt, Maryland, USA
}

(2nd revision)

January 18,2008

Submitted to Quarterly Journal of the Royal Meteorological Society

Corresponding author address: Dr. Xiping Zeng, Mail Code 613.1, NASA/Goddard Space Flight Center, Greenbelt, MD 20771, USA. Email: zeng@agnes.gsfc.nasa.gov 


\section{SUMMARY}

This theoretical study is carried out to investigate the effect of radiation on ice crystal spectrum in the upper troposphere. First, an explicit expression is obtained for the ice crystal growth rate that takes account of radiative and kinetic effects. Second, the expression is used to quantitatively analyze how radiation broadens the ice crystal spectrum and then reveal a new precipitation mechanism in the upper troposphere and the stratosphere. Third, the radiative effect is used to explain the subvisual clouds near the tropopause.

KEYWORDS: Radiation Cirrus Ice Crystal Spectrum 


\section{Introduction}

Tropical precipitation is still a challenge in meteorology (Simpson et al. 1988). It involves multi-scale processes. Small-scale precipitating convection has a propensity to spontaneously organize into coherent structures or cloud clusters (e.g., Ludlam 1980; Moncrieff 2004), and cloud clusters in turn generate cloud shields in their mature and later stages (Zipser 1969; Houze 1977, 1982). Since cloud shields are widespread (or 104$10^{5} \mathrm{~km}^{2}$ in area; Houze 1982; Nesbitt et al. 2000), they modulate atmospheric radiation and thus change large-scale vertical circulations (Houze 1982). As a feedback, large-scale circulations modify clouds and precipitation (Raymond and Zeng 2005). In this multiscale feedback, convective clouds and large-scale circulations are highly coupled through upper-tropospheric cirrus (e.g., Raymond and Zeng 2000).

Theoretically speaking, cloud-resolving models can be used to evaluate the cirrus in the coupling between convective clouds and large-scale circulations. However, recent evaluations of cloud-resolving model simulations showed that the models predicted excessive clouds especially in the upper troposphere (e.g., Petch and Gray 2001; Zeng et al. 2007). Moreover, Starr and Quante (2002) compared the results of cirrus models using bin and bulk representation of microphysical processes. They pointed out that the bulkparameterization model diagnosed a significant population of large ice particles while the bin model was dominated by small ice particles. Those modeling studies suggest that some physical processes be overlooked in the current cloud models.

Recent aircraft observations of ice particles (e.g., Heymsfield 1986; Heymsfield et al. 2006) have provided clues as to the processes that were overlooked in the current cloud 
models. Heymsfield (1986) showed that long-lasting subvisual clouds near the tropical tropopause consisted of ice crystals with small size and low number density. Heymsfield et al. (2006) reported that the maximum measured ice particle diameter increased with air temperature from -70 to $-85^{\circ} \mathrm{C}$ when convection existed below but decreased in the absence of deep convection.

Those modeling and observational issues may involve cloud-radiation interaction (see section 6 for discussion). Previous studies have proposed that radiation affects the diffusional growth of ice crystals (Hall and Pruppacher 1976; Stephens 1983; Hallett 1987; Hallett et al. 2002). Stephens (1983) showed that radiation significantly increases the survival distance of falling ice crystals in an unsaturated environment. He further suggested that the radiative effect on ice crystal growth be introduced in cloud models. However, few current cloud models (Wu et al. 2000) account for it. One possible reason is that the ice crystal growth rate with the effect is expressed implicitly through the ice crystal surface temperature, making it less easy for a cloud model to adopt. Hence, it is imperative to obtain an explicit expression for the ice crystal growth rate first.

Accordingly, the radiative effect is studied in the present paper beginning with an explicit expression for the ice crystal growth rate. The expression is then used to quantitatively analyze how radiation broadens the ice crystal spectrum and finally propose a new precipitation mechanism in the upper troposphere. The paper consists of seven sections. Section 2 describes a theoretical framework for ice crystal growth that takes account of radiative and kinetic effects. Sections 3 and 4 introduce linear and nonlinear models to show how radiation affects ice crystal spectrum, respectively. Section 5 presents a condition for haze aloft. Section 6 uses the present results to explain 
some clouds in the upper troposphere. Section 7 concludes. Except when specified, the paper follows the symbol definition in appendix A.

\section{Theoretical framework}

\subsection{Heat balance of an ice crystal}

The heat balance of an ice crystal in a rarefied atmosphere is represented with the following form (Roach 1976; Hall and Pruppacher 1976; Stephens 1983; Hallett 1987; Hallett et al. 2002):

$$
L_{s} 4 \pi C D F_{\beta}\left[\rho_{s i}\left(T_{s}\right)-\rho_{v}\right] f_{m}=4 \pi C K F_{\alpha}\left(T_{e}-T_{s}\right) f_{Q}+S F_{s}-S \varepsilon_{0} \sigma\left(T_{s}^{4}-\varepsilon_{r} \eta T_{e}^{4}\right)
$$

where $T_{s}$ and $T_{e}$ refer to the ice crystal surface and the environmental air temperature, respectively; $\rho_{v}$ and $\rho_{s i}$ denote the density of the ambient water vapor and saturated water vapor near a crystal, respectively; $S$ denotes crystal surface area; $f_{m}$ and $f_{Q}$ are the ventilation factors expressed in Hall and Pruppacher (1976). Other factors are:

(1) $C$ - the stationary diffusion shape factor that accounts for the influence of crystal shape on the water vapor field around a crystal. For a spherical crystal of radius $r, C=r$.

(2) $D$ - the diffusivity of water vapor in air that is described by (Hall and Pruppacher 1976)

$$
D=2.11 \times 10^{-5}\left(T / T_{0}\right)^{1.94}\left(p_{0} / p\right) \quad\left[\mathrm{m}^{2} \mathrm{~s}^{-1}\right]
$$

(3) $K$ - the coefficient of thermal conductivity of dry air that is calculated with the formula of Beard and Pruppacher (1971)

$$
K=\left[2.38+0.00703\left(T-T_{0}\right)\right] \times 10^{-2} \quad\left[\mathrm{~J} \mathrm{~m}^{-1} \mathrm{~s}^{-1} \mathrm{~K}^{-1}\right] \text {, }
$$

which is independent of air pressure even when air pressure is low (N. Fukuta 2004, personal communication). 
(4) $F_{\beta}-$ a factor that accounts for the kinetic effect on water vapor diffusion. It is expressed as (Hall and Pruppacher 1976)

$$
F_{\beta}=r^{*} /\left(r^{*}+l_{m}^{*}\right)
$$

where

$$
\begin{aligned}
& r^{*}=S / 4 \pi C \\
& l_{m}^{*}=\left(\frac{2 \pi}{R_{v} T_{s}}\right)^{1 / 2} \frac{D f_{m}}{2 \beta(2-\beta)^{-1}} .
\end{aligned}
$$

(5) $F_{\alpha}-$ a factor that accounts for kinetic effect on heat diffusion. It takes the form (Fukuta and Walter 1970; Carstens 1972; Carstens et al. 1974; Hall and Pruppacher 1976; Pruppacher and Klett 1997)

$$
F_{\alpha}=r^{*} /\left(r^{*}+l_{Q}^{*}\right)
$$

where

$$
l_{Q}^{*}=\left(\frac{2 \pi}{R_{d} T_{s}}\right)^{1 / 2} \frac{K f_{Q}}{\rho_{a} C_{p} 2 \alpha(2-\alpha)^{-1}} .
$$

The factors $2 \beta(2-\beta)^{-1}$ and $2 \alpha(2-\alpha)^{-1}$ in (2.5) and (2.7) are referred to as the apparent deposition and thermal accommodation coefficients, respectively (Chapman and Cowling 1970; Fukuta $2004^{1}$ ). Based on experimental measurements (e.g., Fukuta and Walter 1970; Shaw and Lamb 1999), the thermal accommodation coefficient $\alpha=0.7$, and the

\footnotetext{
${ }^{1}$ In a copy of his manuscript "Advanced Cloud Physics" received in 2004, Prof. N. Fukuta used the representative deposition coefficient and thermal accommodation coefficient to name the two factors, respectively.
} 
deposition coefficient $\beta=0.04 \exp \left[-\left(T-T_{0}\right) / 85\right]$ that fits the data of Fukuta and Walter (1970) when $\mathrm{T}>-85^{\circ} \mathrm{C}$.

(6) $F_{s}-$ the solar flux absorbed by an ice crystal.

(7) $\varepsilon_{0}$ - the bulk absorption efficiency of an ice crystal for blackbody radiation. It is defined as the ratio of the infrared flux absorbed by a crystal to the radiative flux emitted by a blackbody at the crystal surface temperature. Based on the results of Stephens (1983), the efficiency is approximated with $\varepsilon_{0}=0.9\left[1-\exp \left(-r^{*} / 6\right)\right]$ for a crystal surface temperature of $T_{r e f}=261 \mathrm{~K}$, where $r^{*}$ is measured in micrometers. The Wien displacement law shows that the wavelength of peak emission for a blackbody is proportional to the reciprocal of temperature. Since radiation absorption depends on the dimensionless size parameter $2 \pi r^{*} / \lambda$ where $\lambda$ is radiation wavelength, the bulk absorption efficiency at temperature $T$ is approximated with

$$
\varepsilon_{0}\left(r^{*}, T\right)=0.9\left[1-\exp \left(-\operatorname{Tr}^{*} / 6 T_{r e f}\right)\right] .
$$

(8) $\varepsilon_{r}$-the relative bulk absorption efficiency of an ice crystal for infrared radiation. It is defined as $\varepsilon / \varepsilon_{0}$ where $\varepsilon$ represents the ratio of the infrared flux absorbed by a crystal to the infrared flux incident on the crystal. Let $T_{\min }$ and $T_{\max }$ denote the minimum and maximum temperature in the atmosphere and its underlying surface. The radiation emitted by a blackbody at temperature $T_{\min }$ and $T_{\max }$ may impinge on a crystal as two extreme limits. Hence,

$$
\frac{\varepsilon_{0}\left(r^{*}, T_{\min }\right)}{\varepsilon_{0}\left(r^{*}, T\right)}<\varepsilon_{r}\left(r^{*}, T\right)<\frac{\varepsilon_{0}\left(r^{*}, T_{\max }\right)}{\varepsilon_{0}\left(r^{*}, T\right)},
$$

which becomes

$$
T_{\min } / T<\varepsilon_{r}<T_{\max } / T
$$


with the aid of (2.8). Obviously, $\varepsilon_{r}$ varies around one. For the sake of simplicity, $\varepsilon_{r}=1$ is assumed except in section 5 .

(9) $\eta$-the ratio of the infrared flux incident on crystal surface to the radiative flux emitted by a blackbody at the environmental air temperature. It is approximated with

$$
\eta=\frac{F^{+}+F^{-}}{2 \sigma T_{e}^{4}},
$$

where $F^{+}$and $F$ represent the upward and downward fluxes of infrared radiation, respectively. Given a vertical distribution of atmospheric variables, the fluxes and thus $\eta$ are determined with radiative processes (e.g., Fu and Liou 1992; Chou et al. 1995).

\subsection{An explicit expression for ice crystal growth rate}

The growth rate of an ice crystal is expressed in terms of ice crystal surface temperature $T_{s}$ (e.g., Hall and Pruppacher 1976). That is

$$
\frac{d m}{d t}=4 \pi C D F_{\beta}\left[\rho_{v}-\rho_{s i}\left(T_{s}\right)\right] f_{m}
$$

where $m$ is crystal mass. Next, $(2.1)$ is used to eliminate $T_{s}$ in (2.10) to derive an explicit expression for $d m / d t$.

To simplify the explicit expression and its derivation, a critical case of an ice crystal in a specific environment is considered first. In the environment, air temperature is still $T_{e}$, but humidity is so specific that the crystal does not grow or shrink. Let the symbol $T_{s c}$ denote the crystal surface temperature, and $e_{c}$ and $H_{i c}$ denote the partial water vapor 
pressure and relative humidity in the critical case ${ }^{2}$, respectively. Thus, the crystal in the critical case has a heat balance between radiative cooling and conductive heating. That is

$$
S \varepsilon_{0} \sigma\left(T_{s c}^{4}-\varepsilon_{r} \eta T_{e}^{4}\right)-S F_{s}=4 \pi C K F_{\alpha}\left(T_{e}-T_{s c}\right) f_{Q} .
$$

Since water vapor is spatially uniform in the critical case,

$$
\rho_{s i}\left(T_{s c}\right)=e_{c}\left(R_{v} T_{e}\right)^{-1} .
$$

After introducing the symbol

$$
\gamma \equiv\left(T_{e}-T_{s c}\right) / T_{e}
$$

and the approximation

$$
T_{s c}^{4} \approx T_{e}^{4}+4 T_{e}^{3}\left(T_{s c}-T_{e}\right),
$$

(2.11) is solved with

$$
\gamma=\frac{\chi}{4(1+\chi)}\left(1-\varepsilon_{r} \eta-\frac{F_{s}}{\varepsilon_{0} \sigma T_{e}^{4}}\right)
$$

where the symbol ${ }^{3}$

$$
\chi=\frac{S \varepsilon_{0} \sigma T_{e}^{3}}{\pi C K F_{\alpha} f_{Q}} .
$$

Using (2.12) and the ideal gas law for water vapor, the critical relative humidity $H_{i c}=e_{c} / E_{s i}\left(T_{e}\right)$ is rewritten as

${ }^{2}$ The critical relative humidity $H_{i c}$ is different from the effective radiative relative humidity defined by Hall and Pruppacher (1976). The latter is associated not only with the radiative effect on ice crystal growth but also the diffusivity of water vapor in air.

${ }^{3}$ The symbol $\chi$, as introduced in (2.20), represents the ratio of the infrared flux to the conductive heat flux from air to an ice crystal, where both air and ice crystal are treated as black bodies. 


$$
H_{i c}=\frac{T_{e} E_{s i}\left(T_{s c}\right)}{T_{s c} E_{s i}\left(T_{e}\right)} .
$$

This expression is changed further to

$$
H_{i c}=\frac{1}{1-\gamma}\left(1-\frac{L_{s} \gamma}{R_{v} T_{e}}\right)
$$

with the aid of (2.13), (2.15) and the following approximation

$$
\frac{E_{s i}(T)}{E_{s i}\left(T_{e}\right)} \approx \frac{L_{s}}{R_{v}} \frac{T-T_{e}}{T_{e}^{2}}+1
$$

that is obtained from the Clausius-Clapeyron equation while $T$ is close to $T_{e}$.

Starting with expression (2.18) for the critical case, an explicit expression is derived next for the growth rate of an ice crystal in a general environment. Subtracting (2.11) from (2.1) yields

$$
L_{s} 4 \pi C D F_{\beta}\left[\rho_{s i}\left(T_{s}\right)-\frac{\left(e T_{s c} / T_{e}\right)}{R_{v} T_{s c}}\right] f_{m}=4 \pi C K(1+\chi) F_{\alpha}\left(T_{s c}-T_{s}\right) f_{Q},
$$

using (2.14), (2.16) and the ideal gas law for water vapor. Equation (2.20) is still equivalent to (2.1) because the known variable $T_{s c}$ always satisfies (2.11).

Fortunately, (2.20) is formally equivalent to the energy equation of the same crystal in an environment that is characterized as having an air temperature $T_{s c}$, partial water vapor pressure $\left(e T_{s c} / T_{e}\right)$, coefficient of thermal conductivity $K(1+\chi)$, and no radiative effect. Deriving the explicit expression for ice crystal growth rate [or the left side of (2.20)] just as done in many textbooks on cloud physics (e.g., Rogers and Yau 1989) gives

$$
\frac{d m}{d t}=\frac{4 \pi C}{A\left(T_{s c}\right)+B\left(T_{s c}\right)}\left[\frac{\left(e T_{s c} / T_{e}\right)}{E_{s i}\left(T_{s c}\right)}-1\right]
$$

where 


$$
\begin{aligned}
& A(T)=\frac{L_{s}}{F_{\alpha} f_{Q} K(1+\chi) T}\left(\frac{L_{s}}{R_{v} T}-1\right) \\
& B(T)=\frac{R_{v} T}{F_{\beta} f_{m} D E_{s i}(T)} .
\end{aligned}
$$

To remove $T_{s c}$ from $(2.21), A\left(T_{s c}\right)$ and $B\left(T_{s c}\right)$ are changed to

$$
\begin{aligned}
& A\left(T_{s c}\right) \approx A\left(T_{e}\right) \frac{T_{e}^{2}}{T_{s c}^{2}} \\
& B\left(T_{s c}\right) \approx B\left(T_{e}\right) \frac{T_{e} E_{s i}\left(T_{e}\right)}{T_{s c} E_{s i}\left(T_{s c}\right)}
\end{aligned}
$$

with the aid of (2.2) and $\left|T_{s c}-T_{e}\right|<<T_{e}$. Substituting (2.17) and the preceding two approximations into (2.21) yields an explicit expression for the growth rate of an ice crystal in a general environment. That is

$$
\frac{d m}{d t}=\frac{4 \pi C\left(H_{i}-H_{i c}\right)}{(1-\gamma)^{2}\left[H_{i c} A\left(T_{e}\right)+B\left(T_{e}\right)\right]},
$$

which does not involve $T_{s}$. Expression (2.24) shows that an ice crystal grows when relative humidity $H_{i}>H_{i c}$ and shrinks when $H_{i}<H_{i c}$. If $\varepsilon_{r} \eta=1$ and $F_{s}=0, H_{i c}=100 \%$, which reduces the expression into the traditional one without the radiative effect (e.g., Rogers and Yau 1989).

Infrared and solar radiation effect $H_{i c}$. Due to $\gamma<<1$ and $\chi<<1,(2.15)$ and (2.18) show that

$$
\left(1-H_{i c}\right) \propto\left(1-\varepsilon_{r} \eta-\frac{F_{s}}{\varepsilon_{0} \sigma T_{e}^{4}}\right) .
$$

Since infrared absorption of ice crystals is more important than solar absorption, the effect of solar radiation on ice crystal growth is neglected in the paper (i.e., $\left.F_{s} / \varepsilon_{0} \sigma T_{e}^{4}=0\right)$. Next, $H_{i c}$ is discussed by dividing $\left(1-H_{i c}\right)$ into two factors: $\left(1-\varepsilon_{r} \eta\right)$ and 
$\left(1-H_{i c}\right) /\left(1-\varepsilon_{r} \eta\right)$. The former factor involves radiative processes and the latter one depends on crystal size, shape and other non-radiative variables.

\subsection{Infrared ratio $\eta$}

The infrared ratio $\eta$ approximately measures the relative magnitude of inward and outward infrared fluxes on the surface of an ice crystal. When $\varepsilon_{r} \eta<1, H_{i c}<100 \%$ and thus radiation enhances crystal growth. Otherwise, $H_{i c}>100 \%$ and radiation benefits crystal shrinkage. Since $\eta$ varies with the variables on air temperature, water vapor and clouds, the following example is presented to show how $\eta$ varies with height through the variables.

Consider the tropical atmosphere observed during the Kwajalein Experiment (KWAJEX) that was conducted around Kwajalein Atoll from July 23 through September 15, 1999. Figure 1 shows the mean air temperature and mixing ratio of water vapor versus pressure. Air temperature decreases considerably with pressure from 300 to 100 hpa.

Given a cloud distribution in the atmosphere, $\eta$ is calculated using (2.9) and the code for radiative transfer (Chou et al. 1995). Its results are displayed against pressure in Fig. 2 for three given cloud distributions. When the sky is clear, $\eta>1$ above the 250 hpa level and $\eta<1$ below that height. The factor $\eta$ reaches a minimum of 0.89 at $428 \mathrm{hpa}$ and a maximum of 1.8 at 100 hpa. Comparing Figs. 1 and 2 shows that the height for the maximum $\eta$ corresponds to the height for the minimum temperature. Hence, $\eta$ is largest at the tropopause. 
Consider a thick stratiform cloud with the top at $600 \mathrm{hpa}$. Since the cloud behaves like a black body, $\eta$ is close to one in the cloud. Above the cloud, $\eta$ varies with height similar to in a clear sky. Nevertheless, $\eta$ is smaller than that for a clear sky, because the upward infrared flux decreases with decreasing the underlying-blackbody temperature from the sea surface temperature to cloud top temperature.

Suppose the cloud top increases from 600 to $200 \mathrm{hpa}$. The cloud is so thick that it blocks the upward infrared radiation from the sea surface and the air below. Thus, it radiates upward as a black body but at a relatively low cloud top temperature. Hence, high cirrus significantly decreases $\eta$ and therefore greatly enhances the growth of ice crystals above itself.

\subsection{The effect of crystal size and shape on $H_{i c}$}

Once $\varepsilon_{r} \eta$ deviates from one, the radiative effect on ice crystal growth is proportional to a ratio of $\left(1-H_{i c}\right) /\left(1-\varepsilon_{r} \eta\right)$. The ratio depends on crystal size and shape as well as air temperature and pressure, which is discussed next.

Consider a spherical crystal. Given $T$ and $p,\left(1-H_{i c}\right) /\left(1-\varepsilon_{r} \eta\right)$ varies with radius. Figure 3 displays the ratio versus radius when $f_{Q}=1$. The ratio increases with crystal size. Hence, $H_{i c}$ decreases and increases with crystal size when $\eta<1$ and $\eta>1$, respectively, because $\varepsilon_{r}$ is close to one (see section 5 for the effect of $\varepsilon_{r}$ on $H_{i c}$ ).

The ratio $\left(1-H_{i c}\right) /\left(1-\varepsilon_{r} \eta\right)$ also varies with height through air temperature and pressure. As shown in Fig. 3, the ratio increases considerably with decreasing height. It, for example, increases from 59 to $81 \%$ at $r^{*}=1000 \mu \mathrm{m}$ when $(T, p)$ changes from $\left(-80^{\circ} \mathrm{C}, 100\right.$ hpa) to $\left(-6^{\circ} \mathrm{C}, 500 \mathrm{hpa}\right)$. 
Figure 3 is suitable for non-spherical crystals, too. A columnar-shaped crystal is modeled by a prolate spheroid of semi-major and minor axis lengths $a$ and $b$, for which (Pruppacher and Klett 1997)

$$
\left.C=\sqrt{a^{2}-b^{2}} / \ln \left[\left(a+\sqrt{a^{2}-b^{2}}\right) / b\right)\right\rfloor .
$$

A plate-like ice crystal is modeled by an oblate spheroid of semi-major and minor axis lengths $a$ and $b$, for which (Pruppacher and Klett 1997)

$$
C=\sqrt{a^{2}-b^{2}} / \arcsin \sqrt{1-b^{2} / a^{2}} .
$$

After defining $r^{*}$ in (2.4) and using it as the horizontal axis, Fig. 3 displays as $H_{i c}$ versus $r^{*}$ for non-spherical crystals.

\section{Two unsteady modes}

\subsection{A linear model}

Expression (2.24) is used to analyze how radiation impacts ice crystal spectrum for a given relative humidity. Consider an ice crystal that deviates about its initial state; then (2.24) is linearized as

$$
d s / d t=k\left(s_{0}\right)\left[H_{i}-H_{i c}\left(s_{0}\right)\right]+\left(s-s_{0}\right) / \tau\left(s_{0}\right),
$$

where subscript " 0 " indicates the initial state and

$$
\begin{aligned}
& s=4 \pi r^{*_{2}} \\
& \tau(s)=-\left[k(s) d H_{i c} / d s\right]^{-1} \\
& k(s)=\frac{4 \pi C}{(1-\gamma)^{2}\left[H_{i c} A\left(T_{e}\right)+B\left(T_{e}\right)\right]}\left(\frac{d m}{d s}\right)^{-1} .
\end{aligned}
$$

Buoyancy waves and underlying clouds modulate $H_{i}$ and $H_{i c}$, respectively. Without loss of generality, suppose that 


$$
H_{i}-H_{i c}\left(s_{0}\right)=\Delta H_{i 0}+A_{H} \sin (\omega t)
$$

where the forcing parameters $\Delta H_{i 0}, A_{H}$ and $\omega$ are constant. Substituting (3.5) into (3.1) and then solving the resulting equation yields

$$
\begin{aligned}
s-s_{0}= & -\frac{k A_{H}}{\tau^{-2}+\omega^{2}}\left[\tau^{-1} \sin (\omega t)+\omega(\cos (\omega t)-1)\right] \\
& +k \Delta H_{i 0} \tau\left(e^{t / \tau}-1\right)+\frac{k A_{H}(\omega \tau)}{1+(\omega \tau)^{2}} \tau\left(e^{t / \tau}-1\right)
\end{aligned}
$$

where $k$ and $\tau$ take their values at $s=s_{0}$. As shown in the preceding equation, crystal growth accelerates when $A_{H}>0$ and $\Delta H_{i 0}>0$, and shrinkage is enhanced when $A_{H}<0$ and $\Delta H_{i 0}<0$. These two unsteady modes broaden and narrow the ice crystal spectrum when $\eta<1$ and $\eta>1$, respectively, which is detailed in section 4.2 .

The third term on the right hand side of (3.6) represents the bulk ice crystal growth in a period due to a buoyancy wave or underlying-cloud variation. It has a factor of $(\omega \tau) /\left[1+(\omega \tau)^{2}\right]$, which reaches a maximum at $\omega \tau=1$. For a given $\tau$, the factor (or the spectrum broadening) is directly proportional to the forcing period $2 \pi / \omega$ when $\omega \tau \gg>1$ and inversely proportional to the forcing period when $\omega \tau<<1$.

\subsection{Timescale for spectrum broadening}

The second and third terms on the right hand side of (3.6) share a common factor of $\tau\left(e^{t / \tau}-1\right)$ that is inversely proportional to $\tau$. For solid spherical crystals without involving radiation, $\tau \rightarrow \infty$, which results in $\tau\left(e^{t / \tau}-1\right)=0$. Hence, an ice crystal spectrum with $|\tau|<\infty$ is broadened or narrowed with respect to a spectrum with $\tau \rightarrow \infty$. In brief, $\tau$ measures the spectrum broadening (or narrowing) due to radiation. 
The timescale $\tau$ relies on $\varepsilon_{r}, \eta$, crystal size, shape, air temperature and pressure. As shown by (2.15), (2.18) and (3.3), $\tau$ is almost inversely proportional to $1-\varepsilon_{r} \eta$. Thus, the next discussions address $\tau\left(1-\varepsilon_{r} \eta\right)$ versus crystal size and shape first and then air temperature and pressure.

Figure 4 displays $\tau\left(1-\varepsilon_{r} \eta\right)$ versus $r^{*}$ for five crystal shapes when the air temperature and pressure equal $-80^{\circ} \mathrm{C}$ and $100 \mathrm{hpa}$, respectively. Using ice crystal parameters from observations, the timescale is calculated with (3.3), which is detailed next.

- A graupel particle is treated as a sphere with a bulk ice density of $0.9 \mathrm{~g} \mathrm{~kg}^{-1}$ and ventilation factors $f_{m}$ and $f_{Q}$ are assumed to be ones. This crystal shape is so simple that $\tau$ versus $r^{*}$ is easily understood with (3.2)-(3.4). Thus, the shape is discussed here as a reference for other shapes. Figure 4 displays the timescale $\tau\left(1-\varepsilon_{r} \eta\right)$ of graupel against $r^{*}$. The timescale decreases and increases with $r^{*}$ when $r^{*}$ is small and large, respectively. It reaches a minimum of 3.5 days at $r^{*}=5 \mu \mathrm{m}$.

- A bullet-shaped crystal is modeled with a prolate spheroid of semi-major and minor axis lengths $a$ and $b$, for which the surface area

$$
S=2 \pi b\left(b+\frac{a^{2}}{\sqrt{a^{2}-b^{2}}} \arcsin \frac{\sqrt{a^{2}-b^{2}}}{a}\right) .
$$

The expressions for the mass and terminal velocity of bullets come from Heymsfield and Iaquinta (2000); the expression for the dimensional relationship from Heymsfield (1972); and the expressions for the ventilation factors from Hall and Pruppacher (1976). Using those expressions, the timescale for single bullets is calculated and shown against $r^{*}$ in 
Fig. 4. The curves of timescale versus $r^{*}$ are not smooth because the curves for crystal parameters (e.g., mass, dimensional relationship) versus size are not smooth either. Generally speaking, the timescale curve for single bullets is similar to that for graupel except that $\tau\left(1-\varepsilon_{r} \eta\right)$ reaches a minimum of 3.3 days at $r^{*}=12 \mu \mathrm{m}$.

- A plate-like crystal is modeled with an oblate spheroid of semi-major and minor axis lengths $a$ and $b$, for which

$$
S=2 \pi a\left(a+\frac{b^{2}}{\sqrt{a^{2}-b^{2}}} \ln \frac{\sqrt{a^{2}-b^{2}}+a}{b}\right) .
$$

The mass of the plates with dendritic extensions is calculated with the bulk density of Heymsfield (1972). The dimensional relationship of the plates is determined with the observations of Auer and Veal (1970). The terminal velocity is calculated with the expression of Heymsfield (1972). With those relationships, the timescale for plate-like crystals is calculated and shown against $r^{*}$ in Fig. 4. In contrast to graupel, plate-like crystals have a similar timescale versus $r^{*}$ except that $\tau\left(1-\varepsilon_{r} \eta\right)$ reaches a minimum of 2.5 days at $r^{*}=8.9 \mu \mathrm{m}$ when $r^{*}<200 \mu \mathrm{m}$. The decrease in timescale at $r^{*}=200 \mu \mathrm{m}$ coincides with the emergence of dendritic extensions at that size.

- A column-like crystal is modeled with a prolate spheroid. The expressions for the mass and terminal velocity of columns come from Heymsfield and Iaquinta (2000), and that for the dimensional relationship from Auer and Veal (1970) and Heymsfield (1972). Figure 4 displays the timescale for column-like crystals against $r^{*}$ and shows that $\tau\left(1-\varepsilon_{r} \eta\right)$ is close to 3.6 days when $r^{*}$ is between 10 to $60 \mu \mathrm{m}$. 
- A rosette crystal consists of bullets. It is modeled approximately with a sphere. In the present paper, rosettes with four bullets are assumed unless specified. The mass and terminal velocity of rosettes are calculated with the formulas of Heymsfield and Iaquinta (2000). Figure 4 also displays the timescale for the rosettes against $r^{*}$ and shows that $\tau(1-$ $\varepsilon_{r} \eta$ ) reaches a minimum of 0.75 day at $r^{*}=44.8 \mu \mathrm{m}$.

The timescale for spectrum broadening, as summarized in Fig. 4, varies with crystal shape. It varies with crystal size similarly for different shapes. Moreover, it varies considerably with height via air temperature and pressure. Figure 5 displays the timescale versus $r^{*}$ when $(T, p)$ equals $\left(-42^{\circ} \mathrm{C}, 250 \mathrm{hpa}\right)$ and $\left(-6^{\circ} \mathrm{C}, 500 \mathrm{hpa}\right)$, respectively. In comparison to Fig. 4, the figure shows that the timescale versus $r^{*}$ is similar at different heights, but the timescale increases significantly with height. These characteristics on the timescale are helpful in understanding the numerical results in the next section.

\section{Numerical simulations}

The preceding linear model deals with the radiative effect while $H_{i}$ is given. Since crystal growth consumes water vapor, $H_{i}$ changes with time. Next, a nonlinear model that uses $H_{i}$ as a prognostic variable is introduced to show the radiative effect in a general framework.

\subsection{A nonlinear model}


Consider an air parcel that moves vertically. Based on the conservation of energy and water, its relative humidity is governed with

$$
\frac{d H_{i}}{d t}=Q_{1}\left(T_{e}\right) w-Q_{2}\left(T_{e}\right) \sum_{j} \frac{d m_{j}}{d t}
$$

where $m_{j}(j=1,2, \ldots)$ denotes the mass of different sized ice crystals and

$$
\begin{aligned}
& Q_{1}(T)=\frac{1}{T}\left[\frac{L_{s} g}{R_{v} C_{p} T}-\frac{g}{R_{d}}\right] \\
& Q_{2}(T)=\rho_{a}\left[\frac{R_{v} T}{E_{s i}(T)}+\frac{R_{d} L_{s}^{2}}{p R_{v} C_{p} T}\right] .
\end{aligned}
$$

The expressions for $Q_{1}$ and $Q_{2}$ are derived similar to that in Rogers and Yau (1989, p106) for water vapor condensation. Since the present crystal growth rate takes account of the radiative effect, this parcel model represents the radiative effect on ice crystal spectrum.

To model the spectrum evolution accurately, (2.24) is changed to

$$
\frac{d a^{2}}{d t}=\frac{4 \pi C\left(H_{i}-H_{i c}\right)}{(1-\gamma)^{2}\left[H_{i c} A(T)+B(T)\right]}\left(\frac{d m}{d a^{2}}\right)^{-1},
$$

which uses $a^{2}$ rather than $a$ as a prognostic variable because the term on the right hand side of (4.4) is almost constant for spherical crystals. In the following numerical simulations, (4.1) and (4.4) are used as prognostic equations and are integrated with 1024 bins.

\subsection{A mechanism for precipitation formation}

Consider a stationary air parcel with $\varepsilon_{r}=1$ and a constant $\eta$. Based on the linear model in the preceding section, the evolution of the ice crystal spectrum is reasoned as follows. Since $\left(1-H_{i c}\right) /(1-\eta)$ increases with crystal size, $H_{i}$ changes so that eventually it lies 
between $H_{i c}$ for small and large crystals, no matter what the initial relative humidity is. When $\eta<1, H_{i}$ is lower than $H_{i c}$ for small crystals but higher than that for large ones. As a result, small crystals shrink due to sublimation with some eventually disappearing. Large crystals grow due to deposition with some of them becoming precipitating crystals. This sublimation-deposition process broadens the ice crystal spectrum, and therefore converts cloud ice to precipitation. In contrast, when $\eta>1, H_{i}$ is higher than $H_{i c}$ for small crystals but lower than that for large ones. As a result, large crystals shrink due to sublimation while small ones grow due to deposition, narrowing the ice crystal spectrum.

To support this reasoning, a default numerical experiment is done to simulate platelike crystal growth in an air parcel near the tropopause. Suppose that $T=-80^{\circ} \mathrm{C}, p=100 \mathrm{hpa}$ and the initial spectrum of ice crystals

$$
d N(a)=c_{1} a e^{-c_{2} a} d a
$$

where $N(a)$ denotes the number of ice crystals with the semi-major axis length less than a. After choosing $c_{1}=1.25 \times 10^{16} \mathrm{~m}^{-5}$ and $c_{2}=0.5 \mu \mathrm{m}^{-1}$, the initial concentration of ice crystals is $5 \times 10^{4} \mathrm{~m}^{-3}$ and the ice water content $9 \times 10^{-6} \mathrm{~g} \mathrm{~m}^{-3}$, close to observations for subvisual clouds (Heymsfield 1986). Given $\eta=0.5$ and the initial relative humidity $H_{i}=100 \%$, the ice crystal spectrum and $H_{i}$ are simulated using the time step $\Delta t=8 \mathrm{~s}$. The modeled $H_{i}$ is displayed against time in Fig. 6. It decreases slowly with time. Meanwhile, the ice water content increases slowly due to water conservation (figure omitted).

Figure 7 shows the evolution of the mass density $d M(a) / d \ln a$ from $t=0$ to 20 days, where $M(a)$ represents the mass of ice crystals with the semi-major axis length less than a. As shown in the figure, small crystals shrink due to sublimation and large ones grow due to deposition. Correspondingly, the median size of ice crystals increases with time. 
Since the small and large crystals grow oppositely, $H_{i}$ lies between the $H_{i c}$ for small and large crystals. Hence, $H_{i}$ is close to the $H_{i c}$ for the median size crystals. The increase in median crystal size corresponds to the decrease in $H_{i}$ shown in Fig. 6.

If no radiation is involved (or $\eta=1$ ), the ice crystal spectrum and $H_{i}$ remain at their initial state. Hence, radiation is responsible for the spectrum broadening shown in Fig. 7.

To show the radiative effect when $\eta>1$, the default experiment is re-done after increasing $\eta$ from 0.5 to 1.5 and using the final spectrum in Fig. 7 as the initial one. Since (4.1) and (4.4) are symmetric with respect to $\eta=1$, it is easily inferred that the spectrum evolution with $\eta=1.5$ reverses that shown in Fig. 7. Hence, large crystals shrink due to sublimation and small ones grow due to deposition when $\eta>1$. In words, radiation narrows the ice crystal spectrum when $\eta>1$.

\subsection{Sensitivity of precipitation formation to height}

The preceding subsection reveals that precipitation can form on a timescale of days at 100 hpa when $\eta<1$. Here, two additional experiments with $\eta=0.5$ are carried out to show that precipitation forms faster at lower heights for the same $\eta$. The first additional experiment uses the same parameters as the default one except for $T=-42^{\circ} \mathrm{C}$ and $p=250$ hpa. In the experiment, a parameter for initial ice crystal spectrum $c_{1}=1.25 \times 10^{18} \mathrm{~m}^{-5}$, which corresponds to an initial crystal concentration of $5 \times 10^{6} \mathrm{~m}^{-3}$ and an ice water content of $9 \times 10^{-4} \mathrm{~g} \mathrm{~m}^{-3}$. The experiment is performed with a time step $\Delta t=0.5 \mathrm{~s}$. Figure 8 displays the evolution of the mass density from $t=0$ to 6 hours, showing that precipitation can form on a timescale of hours at $250 \mathrm{hpa}$, much shorter than that at $100 \mathrm{hpa}$. 
The second additional experiment uses the same parameters as the first additional one except for $T=-6^{\circ} \mathrm{C}$ and $p=500 \mathrm{hpa}$. The experiment was performed with $\Delta t=0.02 \mathrm{~s}$. Figure 9 displays the evolution of the mass density from $t=0$ to 15 minutes, revealing that precipitation can form on a timescale of ten minutes at $p=500$ hpa. In summary, precipitation formation due to radiation is sensitive to height via air temperature and pressure. This sensitivity of precipitation formation to height is a result of the sensitivity of the timescale to height shown in Figs. 4 and 5.

\section{Spectral asymmetry between absorbed and emitted radiation}

Consider an optically-thin cloud in a clear stationary atmosphere. The cloud is so thin that it has almost no effect on atmospheric radiation. Thus, much of the infrared radiation emitted by the underlying surface can reach the crystals in the cloud through the atmospheric thermal infrared window, leading to an obvious spectral asymmetry between crystal-absorbed and emitted radiation (or $\varepsilon_{r} \neq 1$ ). Next, a crystal in the cloud is discussed as an example to show the effect of the spectral asymmetry on $H_{i c}$.

For an ice crystal in the cloud, $\varepsilon_{r}$ is close to its maximum, or

$$
\varepsilon_{r} \approx \varepsilon_{0}\left(r^{*}, T_{u s t}\right) / \varepsilon_{0}\left(r^{*}, T\right)
$$

where $T_{u s t}$ is the underlying surface temperature. Equations (2.8) and (5.1) show that $\varepsilon_{r}>1$ while $T<T_{\text {ust }}$.

The infrared ratio $\eta$, just as shown in Fig. 2, is larger than one in the upper troposphere but smaller than one in the middle and lower troposphere. Consider an ice crystal with an environment of $\eta=1.8, T=-80^{\circ} \mathrm{C}, p=100 \mathrm{hpa}$, and $T_{u s t}=300 \mathrm{~K}$. Using $\varepsilon_{r}$ in (5.1), $H_{i c}$ is calculated and displayed against $r^{*}$ in Fig. 10. Meanwhile, the $H_{i c}$ with $\varepsilon_{r}=1$ is 
displayed in the same figure for comparison. Obviously, $\varepsilon_{r}$ effects $H_{i c}$ considerably when $r^{*}<40 \mu \mathrm{m}$. It changes $H_{i c}$ by $0.2 \%$ at $r^{*}=12 \mu \mathrm{m}$. Since $\varepsilon_{r}$ does not reverse the increase in $H_{i c}$ with increasing $r^{*}$, it affects the sublimation-deposition process only quantitatively while $\eta>1$.

In contrast, consider a crystal with an environment of $\eta=0.9, T=-12^{\circ} \mathrm{C}, p=430 \mathrm{hpa}$, and $T_{u s t}=300 \mathrm{~K}$. Using $\varepsilon_{r}$ in (5.1), $H_{i c}$ is calculated and displayed in Fig. 11. It increases and decreases with $r^{*}$ while $r^{*}$ is smaller and larger than $r_{M A X}^{*}=1.9 \mu \mathrm{m}$, respectively. In other words, $H_{i c}$ reaches a maximum of $H_{i C M A X}=1+6 \times 10^{-6} \%$ at $r^{*}=r_{M A X}^{*}$. When $1<H_{i}<H_{i c M A X}$, there are two equilibrium states [or $H_{i}=H_{i c}\left(r^{*}\right)$ ] for an ice crystal. The equilibrium state with the smaller $r^{*}$ is stable that corresponds to haze aloft. The other equilibrium state with the larger $r^{*}$ is unstable. A crystal at the unstable equilibrium state, once perturbed initially, either shrinks to reach the stable state or grows to become a precipitating crystal.

When many ice crystals coexist under $\eta<1$ and some of them have $H_{i c}$ less than $100 \%$, the sublimation-deposition process works to form precipitation just as in the atmosphere with $\varepsilon_{r}=1$. In summary, $\varepsilon_{r}$ cannot change the sublimation-deposition process greatly even while $\eta<1$.

\section{Discussion}

The preceding sections proposed a precipitation mechanism: when $\eta<1$, the sublimation-deposition process due to radiation converts cloud ice to precipitating ice that in turn sediments to dehumidify air. This process, different from particle 
sedimentation, can work as a source of large particles near cloud top. Next, cloud-top observations are discussed to infer the existence of the process.

\subsection{Subvisual clouds}

Heymsfield (1986) observed the microphysical structure of subvisual clouds near the tropical tropopause and showed that the clouds consist of ice particles with a mean diameter of $2.5 \mu \mathrm{m}$ and number density of less than $5 \times 10^{4} \mathrm{~m}^{-3}$. The subvisual clouds are common, but not pervasive, in the Tropics and subtropics (Winker and Trepte 1998). They are separated from cirrus below but tend to occur with thick cirrus below (Heymsfield 1986; Winker and Trepte 1998). They can also persist for weeks or months in clear skies in the equatorial regions (Lynch and Sassen 2002). These observations match the characteristics of the radiative effect, which is shown next.

In the Tropics, convective clouds drive large-scale upward motion in the troposphere (Riehl and Malkus 1958). Thus, horizontal water advection cannot generate subvisual clouds near the tropopause. On the other side, local vertical circulations (including turbulent motions) transport water downgradient near the tropopause. As a result, water accumulates near the tropopause, and thus pervasive clouds are expected there.

The sublimation-deposition process due to radiation can break the pervasive clouds into subvisual clouds. Since high cirrus leads to $\eta<1$ near the tropopause (see Fig. 2), the process, accompanied with high cirrus below, can convert small ice crystals to large ones that in turn sediment, serving as a water sink near the tropopause. Owing to the maximum $\eta$ and minimum $T$ at the tropopause, the conversion of cloud ice to precipitation is the least efficient there. As a result, the process can separate subvisual clouds from cirrus 
below. With the aid of the sublimation-deposition process, high cirrus can enlarge the crystals near the tropopause that in turn radiate upwards strongly, which resembles the satellite-observed positive correlation between subvisual clouds and the thick cirrus below.

In the absence of high cirrus, $\eta>1$ near the tropopause and large crystals shrink there, which can explain the small size of ice crystals in subvisual clouds. Since ice crystals shrink on a timescale of 10 days (see Figs. 4 and 7), subvisual clouds can last for weeks or months in clear skies. In summary, the observations of subvisual clouds match the characteristics of the radiative effect, which suggests that the sublimation-deposition process exist near the tropopause.

\subsection{Vertical distributions of ice crystal spectrum}

Heymsfield et al. (2006) observed large crystals with a scale up to $10^{3} \mu \mathrm{m}$ at $-85^{\circ} \mathrm{C}$. They reported that the maximum measured ice particle diameter increased from -70 to $85^{\circ} \mathrm{C}$ when convection existed below. That observational phenomenon, involving in the origin of large crystals right below the tropopause, can be explained partly by the radiative effect.

Suppose that convective cells eject ice crystals into the upper troposphere (Heymsfield et al. 2006), which are then blown by the horizontal wind to form a stratiform cloud. The cloud in turn imposes a perturbation on the original vertical $\eta$ distribution. Consider, for example, a stratiform cloud in the atmosphere as discussed in Fig. 1. When the cloud has a constant cloud ice mixing ratio of $0.1 \mathrm{~g} / \mathrm{kg}$ from 132 to $197 \mathrm{hpa}$, it introduces a $\eta$ perturbation as shown in Fig. 12. Since $\eta<1$ near cloud top, radiation can broaden the ice 
crystal spectrum there. In contrast, $\eta>1$ near cloud base, which means that radiation narrows the spectrum there (Stephens 1983). Thus, the radiative effect contributes to the increase in the maximum crystal size with height.

The stratiform cloud discussed in Fig. 12 is optically thick. It imposes a strong perturbation on the original vertical $\eta$ distribution so that $\eta<1$ in a layer. However, an optically thin cloud introduces a weak $\eta$ perturbation so that there is no layer with $\eta<1$ and therefore no layer with spectrum broadening. If convective clouds and their anvils arise below a thin cloud, $\eta$ above the convective clouds is decreased. As a result, $\eta<1$ near the top of the thin cloud and $\eta>1$ near the base, which contributes to an increase in the maximum ice crystal size with height and partly explains the observational phenomenon that the maximum measured ice particle diameter increased from -70 to $85^{\circ} \mathrm{C}$ when convection existed below.

\subsection{Cumulus cloud seeding}

Precipitation initiation in warm cumulus clouds involves the formation of large drops on the scale of cloud lifetimes (e.g., 15 minutes). It requires some process to accelerate the growth of drops at a radius of $\sim 20 \mu \mathrm{m}$ where neither diffusional nor collectional growth is significant. Its current theories (e.g., such as giant salt particles, drop collision due to turbulence, and cloud entrainment) still involve some uncertainties (e.g., Rogers and Yau 1989; Pruppacher and Klett 1997) such that the precipitation initiation is not yet fully understood. Based on the present study, the radiative effect is proposed as a candidate to initiate precipitation in warm cumulus clouds. 
Consider a number of crystals in a blue sky. They form due to gravity waves (or other processes) and some of them have length longer than $\sim 4 \mu \mathrm{m}$. Since $\eta<1$ below the height of the 250 hpa level (see Fig. 2), a part of the crystals can grow to become precipitating ones (see section 5) that fall and then melt below the freezing level. Once the precipitating particles fall into cumulus clouds, they can serve as seeds to initiate frequent collision between drops, which would in turn generate precipitation quickly. From another perspective, radiation can broaden the cloud spectrum near cloud top. Following the same procedure with (2.24) except for variables for liquid water, an explicit expression for the critical relative humidity with respect to water $H_{w c}$ is obtained. Since $H_{w c}$ functions in a similar manner as $H_{i c}$ and $\eta<1$ near cloud top, radiation can broaden the cloud spectrum there and thus contribute partly to precipitation initiation.

In the preceding three cases, the radiative effect is expected to provide large particles near cloud top. Based on the consistence between the characteristics of the radiative effect and the observations of subvisual clouds, it is inferred that the sublimationdeposition process exists in the upper troposphere. Using the present explicit expression for ice crystal growth rate, the radiative effect will be incorporated into a cloud-resolving model for further evaluation.

\section{Conclusions}

This theoretical study addresses the effect of radiation on ice crystal spectrum. From the heat balance equation of an ice crystal, an explicit expression for ice crystal growth rate is obtained that takes account of the radiative effect. The expression introduces three variables: the critical relative humidity $H_{i c}$, the infrared ratio $\eta$, and the relative bulk 
absorption efficiency $\varepsilon_{r}$. An ice crystal grows and shrinks when the relative humidity $H_{i}>H_{i c}$ and $H_{i}<H_{i c}$, respectively.

The critical relative humidity $H_{i c}$ is associated with $\eta$ through an approximately linear relationship between $\left(1-H_{i c}\right)$ and $\left(1-\varepsilon_{r} \eta\right)$ when the effect of solar radiation is ignored. The ratio $\left(1-H_{i c}\right) /\left(1-\varepsilon_{r} \eta\right)$ depends on crystal size and shape as well as air temperature and pressure. Generally speaking, the ratio increases with crystal size. Thus, given $\varepsilon_{r}=1, H_{i c}$ increases and decreases with crystal size when $\eta>1$ and $\eta<1$, respectively.

A linear model is introduced to show how radiation broadens (or narrows) an ice crystal spectrum, where the timescale for spectrum broadening relies on $\varepsilon_{r}, \eta$, crystal size, shape, air temperature and pressure. The timescale is computed using observed parameters of ice crystals. It increases considerably with height through air temperature and pressure. Given $\eta=0.5$, the timescale is measured in minutes, hours and days at the 500, 250 and 100 hpa levels, respectively.

A nonlinear model that uses $H_{i}$ as a prognostic variable is introduced to simulate the evolution of ice crystal spectrum due to radiation. Since $H_{i c}$ varies with crystal size, water is redistributed between ice crystals with different sizes. When $\eta<1$, smaller ice crystals shrink due to sublimation and larger ones grow due to vapor deposition, which results in precipitation formation even when ice water content is small.

The infrared ratio $\eta$ approximately measures the relative magnitude of inward and outward infrared fluxes on the surface of an ice crystal. Owing to the great influence of clouds on $\eta$, the precipitation formation due to radiation is associated with clouds below. Based on the consistence between the characteristics of the radiative effect and the 
observations of subvisual clouds, it is inferred that the precipitation formation due to radiation exists in the upper troposphere.

However, the present parcel model needs to incorporate more physical processes so that its results are quantitatively comparable to field observations. Introducing ice crystal sedimentation, for example, is important in modeling ice crystal spectrum (e.g., Jensen et al. 2007). A spectrally-resolved treatment of radiation (e.g., Stephens 1983; Wu et al. 2000 ) is important in the accurate determination of the infrared ratio $\eta$, the relative bulk absorption efficiency $\varepsilon_{r}$, and the absorbed solar flux $F_{s}$. With the full representation of the physics, the model can simulate optically-thin clouds in contrast to field observations.

Acknowledgements. This study is supported by the Office of Science, U.S. Department of Energy through the Atmospheric Radiation Measurement (ARM) Program. The author appreciates Drs. Andy Heymsfield, Graeme Stephens, David O'C. Starr, Norihiko Fukuta and Ken Beard for clarifying many issues on cloud microphysics and radiation in the course of this study. He thanks Dr. Wei-Kuo Tao for kind support and Steve Lang for improving the manuscript. Special thanks are extended to two anonymous reviewers for their critical yet constructive comments. 


\section{APPENDIX A List of Symbols}

$a / b:$ semi-major/minor axis length

$C:$ stationary diffusion shape factor

$C_{p}:$ specific heat of dry air

$D$ : diffusivity of water vapor

$e:$ partial pressure of water vapor

$e_{c}$ : partial pressure of water vapor in the critical case, see (2.12)

$E_{s i}:$ saturation vapor pressure over ice

$f_{m} / f_{Q}$ : ventilation factor for mass transfer/thermal diffusion

$F_{s}:$ solar flux absorbed by an ice crystal

$F^{+} / F^{*}:$ upward/downward infrared flux

$F_{\alpha} / F_{\beta}$ : factor for the kinetic effect in heat/water vapor diffusion

$g:$ acceleration due to gravity

$H_{i}=e / E_{s i}:$ relative humidity with respect to ice

$H_{i c}:$ critical relative humidity, see (2.18)

$K$ : coefficient of thermal conductivity of dry air

$L_{s}:$ latent heat of sublimation

$m:$ mass of an ice crystal

$M(a)$ : mass of ice crystals with semi-major axis length shorter than $a$

$N(a)$ : number of ice crystals with semi-major axis length shorter than $a$

$r^{*}=S / 4 \pi C$ : equivalent radius of an ice crystal, see (2.4)

$R_{d} / R_{v}:$ gas constant for dry air/water vapor

$p:$ air pressure 
$p_{0}=10^{5}$ pa : reference pressure

$s=4 \pi r^{* 2}$ : area variable for modeling, see (3.2)

$S:$ surface area of an ice crystal

$t:$ time

$T:$ (air) temperature

$T_{e}$ : environmental temperature around an ice crystal

$T_{\mathrm{o}}:$ absolute temperature of ice point

$T_{s}:$ surface temperature of an ice crystal

$T_{s c}$ : crystal surface temperature in the critical case, see (2.11)

$\alpha / \beta$ : thermal accommodation/deposition coefficient

$\gamma$ : relative change of crystal surface temperature due to radiation in the critical case, see

$\varepsilon_{0}$ : bulk absorption efficiency of an ice crystal for blackbody radiation

$\varepsilon_{r}$ : relative bulk absorption efficiency of an ice crystal for infrared radiation

$\sigma:$ the Stefan-Boltzmann constant

$\chi$ : ratio of the infrared flux to the conductive heat flux from air to an ice crystal while

both air and the crystal are treated as black bodies

$\eta$ : infrared ratio, see (2.9)

$\tau$ : timescale for spectral broadening due to radiation, see (3.3)

$\rho_{a} / \rho_{v}:$ density of air/water vapor

$\rho_{s i}$ : saturation density of water vapor over ice

$\Delta t$ : time step for numerical integration 


\section{REFERENCES}

Auer, A. H., and D. L. Veal, 1970: The dimension of ice crystals in natural clouds. $J$. Atmos. Sci., 27, 919-926.

Beard, K. V. and H. R. Pruppacher, 1971: A wind tunnel investigation of the rate of evaporation of small water drops falling at terminal velocity in air. J. Atmos. Sci., 28, $1455-1464$.

Carstens, J. C., 1972: Comments on "Kinetics of hydrometeor growth from a vaporspherical model". J. Atmos. Sci., 29, 588-591.

Carstens, J. C., J. Podzimek, and A. Saad, 1974: On the analysis of the condensational growth of a stationary cloud droplet in the vicinity of activation. J. Atmos. Sci., 31, 592-596.

Chapman, S., and T. G. Cowling, 1970: The mathematical theory of non-uniform gases. Cambridge University Press, pp 422.

Chou, M.-D., W. Ridgway, and M.-H. Yan, 1995: Parameterizations for water vapor IR radiative transfer in both the middle and lower atmosphere. J. Atmos. Sci., 52, 1159-1167.

Fu, Q., and K.-N. Liou, 1992: On the correlated k-distribution method for radiative transfer in nonhomogeneous atmospheres. J. Atmos. Sci., 49, 2153-2170.

Fukuta, N. and L. A. Walter, 1970: Kinetics of hydrometeor growth from a vaporspherical model. J. Atmos. Sci., 27, 1160-1172.

Hall, W. D., and H. R. Pruppacher, 1976: The survival of ice particles falling from cirrus clouds in subsaturated air. J. Atmos. Sci., 33, 1995-2006.

Hallett, J., 1987: Faceted snow crystals. J. Opt. Soc. Am., 4, 581-588. 
Hallett, J., W. P. Arnott, M. P. Bailey, and J. T. Hallett, 2002, Ice crystals in Cirrus.

Cirrus. Lynch, D. K., K. Sassen, D. O'C. Starr and G. Stephens Ed., Oxford University Press, New York. 41-77.

Heymsfield, A., 1972: Ice crystal terminal velocities. J. Atmos. Sci., 29, 1348-1357.

Heymsfield, A. J., 1986: Ice particles observed in a cirriform cloud at $-83^{\circ} \mathrm{C}$ and implications for polar stratospheric clouds. J. Atmos. Sci., 43, 851-855.

Heymsfield, A. J., and J. Iaquinta, 2000: Cirris crystal terminal velocities. J. Atmos. Sci., 57, 916-938.

Heymsfield, A. J., C. Schmitt, and A. Bansemer, 2006: Measurements in low latitude high altitude cirrus. The 12th Conference on Cloud Physics. Madison, WI. July 10-14, 2006.

Houze, R. A., Jr., 1977: Structure and dynamics of a tropical squall-line system observed during GATE. Mon. Wea. Rev., 105, 1540-1567.

Houze, R. A., Jr., 1982: Cloud clusters and large-scale vertical motion in the tropics. $J$. Meteor. Soc. Japan, 60, 396-410.

Jensen, E. J., A. S. Ackerman, and J. A. Smith, 2007: Can overshooting convection dehydrate the tropical tropopause layer? J. Geophy. Res., 112, doi:1029/2006JD007943.

Ludlam, F. H., 1980: Clouds and Storms. Pennsylvania University Press, 405 pp.

Lynch, D. K. and K. Sassen, 2002: Subvisual cirrus. Cirrus, Lynch, D. K., K. Sassen, D. O'C. Starr and G. Stephens Ed., Oxford University Press, New York. 256-264.

Moncrieff, M. W., 2004: Analytic representation of the large-scale organization of tropical convection. J. Atmos. Sci., 61, 1521-1538. 
Nesbitt, S. W., E. J. Zipser, and D. J. Cecil, 2000: A census of precipitation features in the Tropics using TRMM: Radar, ice scattering, and lighting observations. $J$. Climate, 13, 4087-4106.

Petch, J. C., and M. E. B. Gray, 2001: Sensitivity studies using a cloud-resolving model simulation of the tropical west Pacific. Quart. J. Roy. Meteor. Soc., 127, 22872306.

Pruppacher, H. R. and J. D. Klett, 1997: Microphysics of clouds and precipitation. Kluwer, $954 \mathrm{pp}$.

Raymond, D. J. and X. Zeng, 2000: Instability and large-scale circulations in a twocolumn model of the tropical troposphere. Quart. J. Roy. Meteor. Soc., 126, 3117 3135.

Raymond, D. J. and X. Zeng, 2005: Modeling tropical atmospheric convection in the context of the weak temperature gradient approximation. Quart. J. Roy. Meteor. Soc., 131, 1301-1320.

Riehl, H., and J. S. Malkus, 1958: On the heat balance in the equatorial trough zone. Geophysica, 6, 503-538.

Roach, W. T., 1976: On the effect of radiative exchange on the growth by the condensation of a cloud or fog droplet. Quart. J. Roy. Meteor. Soc., 102, 361-372.

Rogers R. R. and M. K. Yau, 1989: A short course in cloud physics. 3rd Edition, Butterworth-Heinemann, Oxford. 290pp.

Shaw, R. A. and D. Lamb, 1999: Experimental determination of the thermal accommodation and condensation coefficients of water. J. Chem. Phys., 111, 10659-10663. 
Simpson, J., R. F. Adler and G. R. North, 1988: A proposed Tropical Rainfall Measuring Mission (TRMM) satellite. Bull. Amer. Meteor. Soc., 69, 278-295.

Starr, D. O., and M. Quante, 2002: Dynamical processes in cirrus clouds. Cirrus, Lynch, D. K., K. Sassen, D. O'C. Starr and G. Stephens Ed., Oxford University Press, New York. 256-264.

Stephens, G. L., 1983: The influence of radiative transfer on the mass and heat budgets of ice crystals falling in the atmosphere. J. Atmos. Sci., 40, 1729-1739.

Winker, D. M. and C. R. Trepte, 1998: Laminar cirrus observed near the tropical tropopause by LITE. Geophy. Res. Lett., 25, 3351-3354.

Wu, T., W. R. Cotton, and W. Y. Y. Cheng, 2000: Radiative effects on the diffusional growth of ice particles in cirrus clouds. J. Atmos. Sci., 57, 2892-2904.

Zeng, X., W.-K. Tao, M. Zhang, C. Peters-Lidard, S. Lang, J. Simpson, S. Kumar, S. Xie, J. L. Eastman, C.-L. Shie and J. V. Geiger, 2007: Evaluating clouds in long-term cloud-resolving model simulations with observational data. J. Atmos. Sci., 64, 4153-4177.

Zipser, E. J., 1969: The role of organized unsaturated convective downdrafts in the structure and rapid decay of an equatorial disturbance. J. Appl. Meteor., 8, 799814. 


\section{Caption}

Figure 1 Vertical profiles of air temperature (thick line) and water vapor mixing ratio (thin line) obtained from averaging 52 days of KWAJEX data.

Figure 2 Infrared ratio $\eta$ as a function of pressure. Thick, thin, and dashed lines represent cases with a clear sky, middle and high clouds, respectively.

Figure 3 Critical relative humidity $H_{i c}$ versus particle radius $r^{*}$, where solid, thin and thick dashed lines represent the results when $(T, p)$ equals $\left(-80^{\circ} \mathrm{C}, 100 \mathrm{hpa}\right),\left(-42^{\circ} \mathrm{C}, 250\right.$ hpa) and $\left(-6^{\circ} \mathrm{C}, 500 \mathrm{hpa}\right)$, respectively.

Figure 4 Timescale $\tau\left(1-\varepsilon_{r} \eta\right)$ versus particle radius $r^{*}$ for five crystal shapes when $(T, p)$ equals $\left(-80^{\circ} \mathrm{C}, 100 \mathrm{hpa}\right)$.

Figure 5 Same as in Fig. 4 except that $(T, p)$ equals $\left(-42^{\circ} \mathrm{C}, 250 \mathrm{hpa}\right)$ and $\left(-6^{\circ} \mathrm{C}, 500 \mathrm{hpa}\right)$ in the upper and lower panels, respectively.

Figure 6 Relative humidity $H_{i}$ versus time in the default experiment where $T=-80^{\circ} \mathrm{C}$, $p=100$ hpa, and $\eta=0.5$.

Figure 7 Evolution of the mass density $d M(a) / d \ln a$ versus the half crystal size $a$ in the default experiment where $T=-80^{\circ} \mathrm{C}$ and $p=100$ hpa. The thick line denotes the initial spectrum, and the time interval between lines is 1 day.

Figure 8 Same as in Fig. 7 except that $T=-42^{\circ} \mathrm{C}, p=250$ hpa and the time interval between lines is 5 minutes.

Figure 9 Same as in Fig. 7 except that $T=-6^{\circ} \mathrm{C}, p=500 \mathrm{hpa}$ and the time interval between lines is 1 minute. 
Figure 10 Critical relative humidity $H_{i c}$ as a function of particle radius $r^{*}$, where $\eta=1.8$, $T=-80^{\circ} \mathrm{C}, p=100 \mathrm{hpa}$, and $T_{u s t}=300 \mathrm{~K}$. The thin and thick lines represent $H_{i c}$ when $\varepsilon_{r}$ equals one and is calculated with (5.1), respectively.

Figure 11 Critical relative humidity $H_{i c}$ as a function of particle radius $r^{*}$, where $\eta=0.9$, $T=-12^{\circ} \mathrm{C}, p=430 \mathrm{hpa}$, and $T_{u s t}=300 \mathrm{~K}$. The thin and thick lines represent $H_{i c}$ when $\varepsilon_{r}$ equals one and is calculated with (5.1), respectively.

Figure 12 Infrared ratio $\eta$ as a function of pressure for two cases. The thick line represents a case for a cloud (denoted by the shaded area). The dashed line represents a case for a clear sky. 


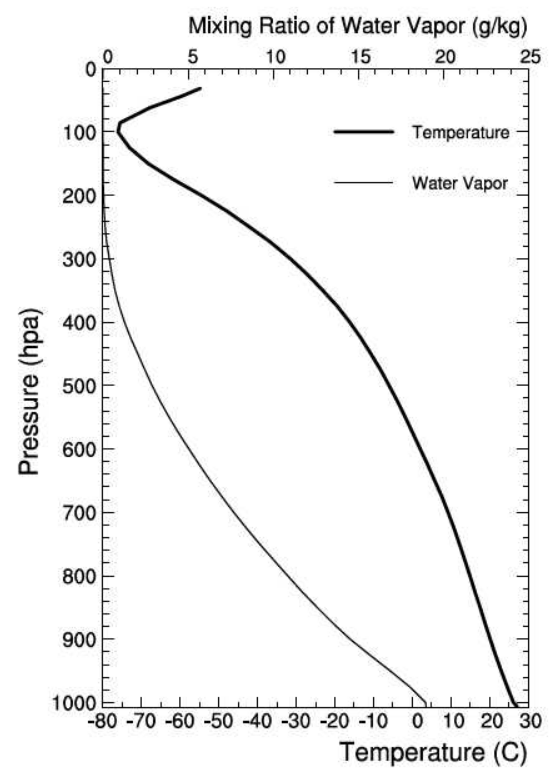

Figure 1 Vertical profiles of air temperature (thick line) and water vapor mixing ratio (thin line) obtained from averaging 52 days of KWAJEX data. 


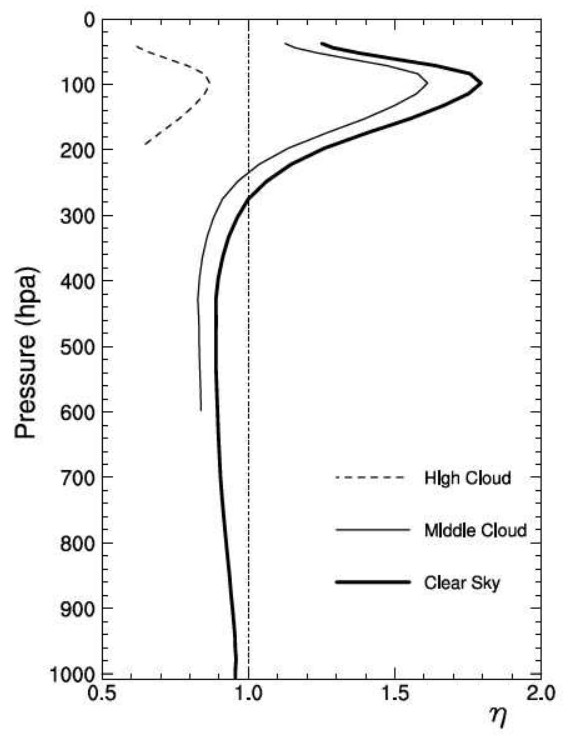

Figure 2 Infrared ratio $\eta$ as a function of pressure. Thick, thin, and dashed lines represent cases with a clear sky, middle and high clouds, respectively. 


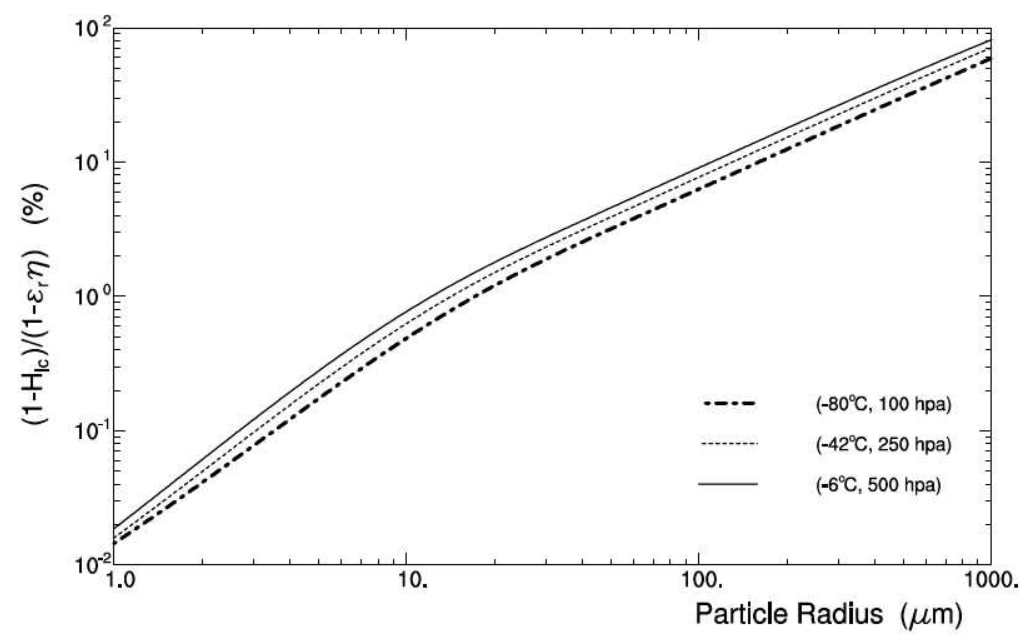

Figure 3 Critical relative humidity $H_{i c}$ versus particle radius $r^{*}$, where solid, thin and thick dashed lines represent the results when $(T, p)$ equals $\left(-80^{\circ} \mathrm{C}, 100 \mathrm{hpa}\right),\left(-42^{\circ} \mathrm{C}, 250\right.$ hpa) and $\left(-6^{\circ} \mathrm{C}, 500 \mathrm{hpa}\right)$, respectively. 


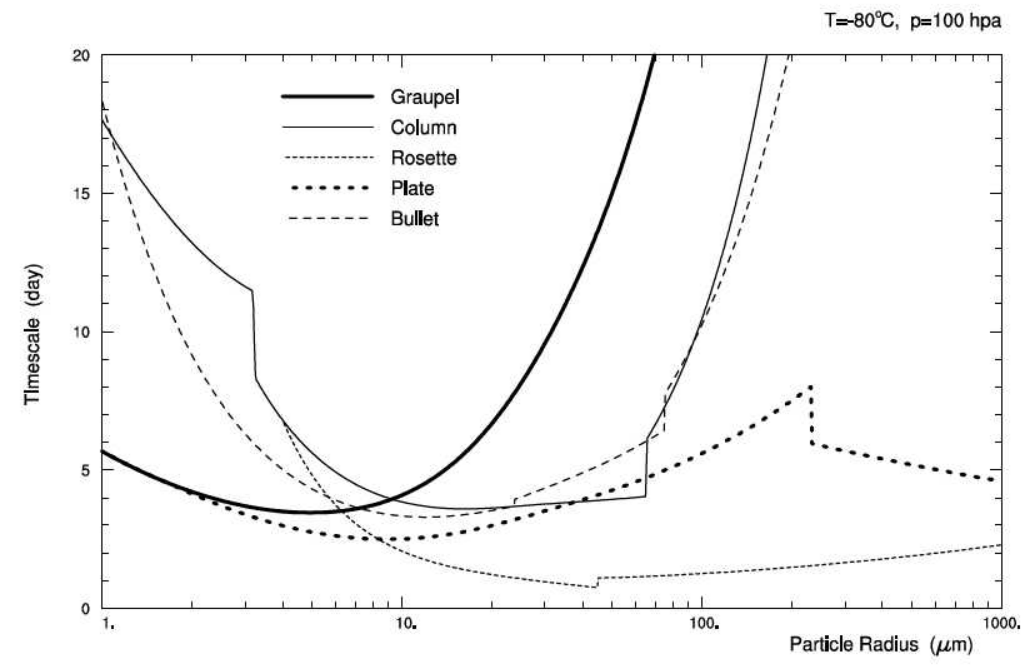

Figure 4 Timescale $\tau\left(1-\varepsilon_{r} \eta\right)$ versus particle radius $r^{*}$ for five crystal shapes when $(T, p)$ equals $\left(-80^{\circ} \mathrm{C}, 100 \mathrm{hpa}\right)$. 

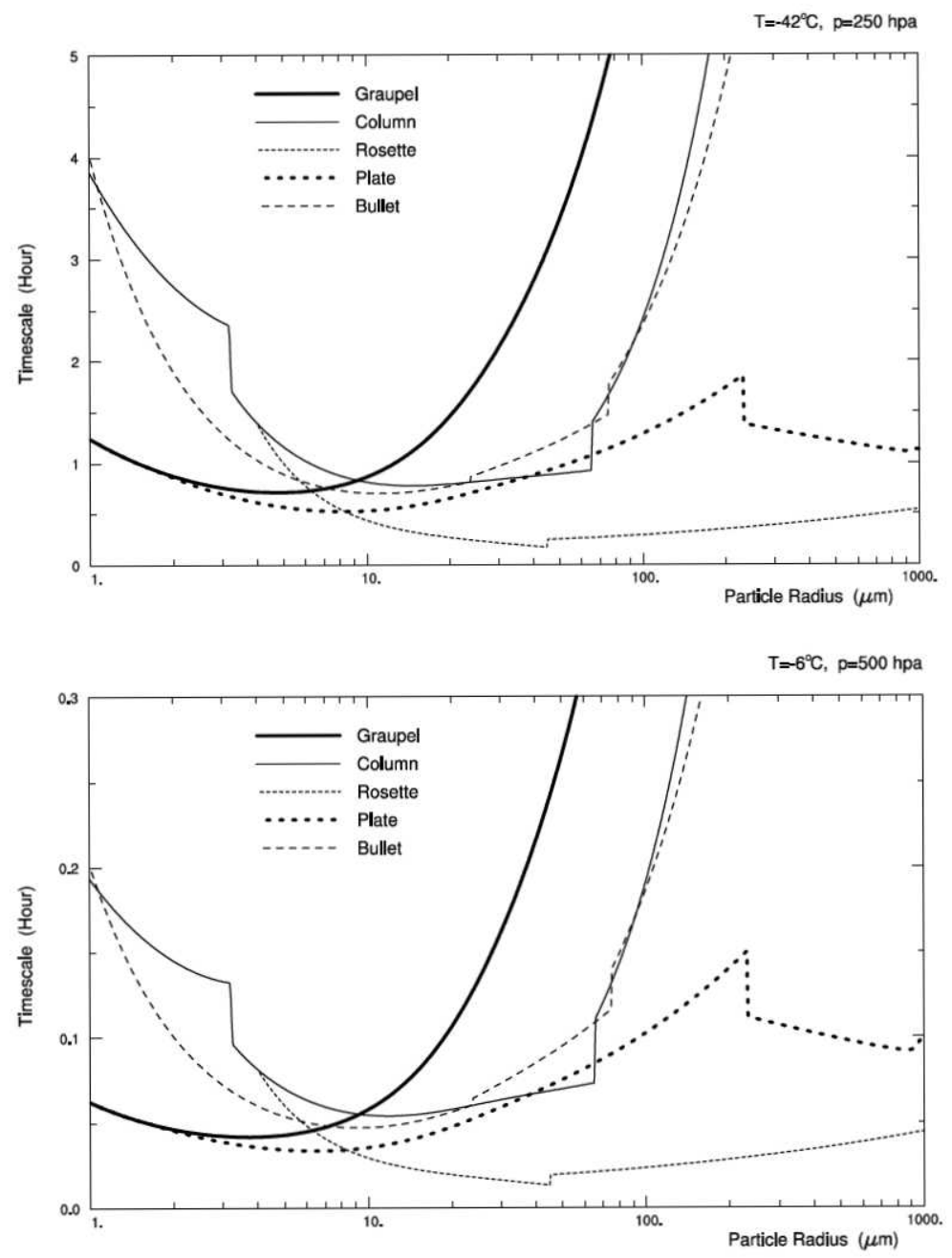

Figure 5 Same as in Fig. 4 except that $(T, p)$ equals $\left(-42^{\circ} \mathrm{C}, 250 \mathrm{hpa}\right)$ and $\left(-6^{\circ} \mathrm{C}, 500 \mathrm{hpa}\right)$ in the upper and lower panels, respectively. 


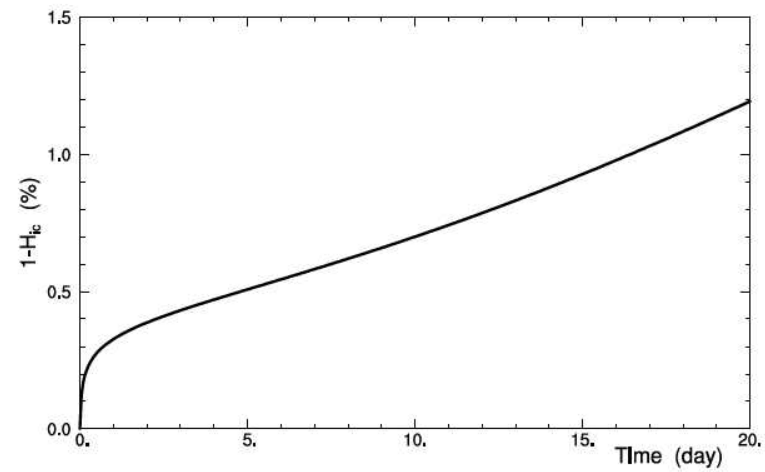

Figure 6 Relative humidity $H_{i}$ versus time in the default experiment where $T=-80^{\circ} \mathrm{C}$, $p=100$ hpa, and $\eta=0.5$. 


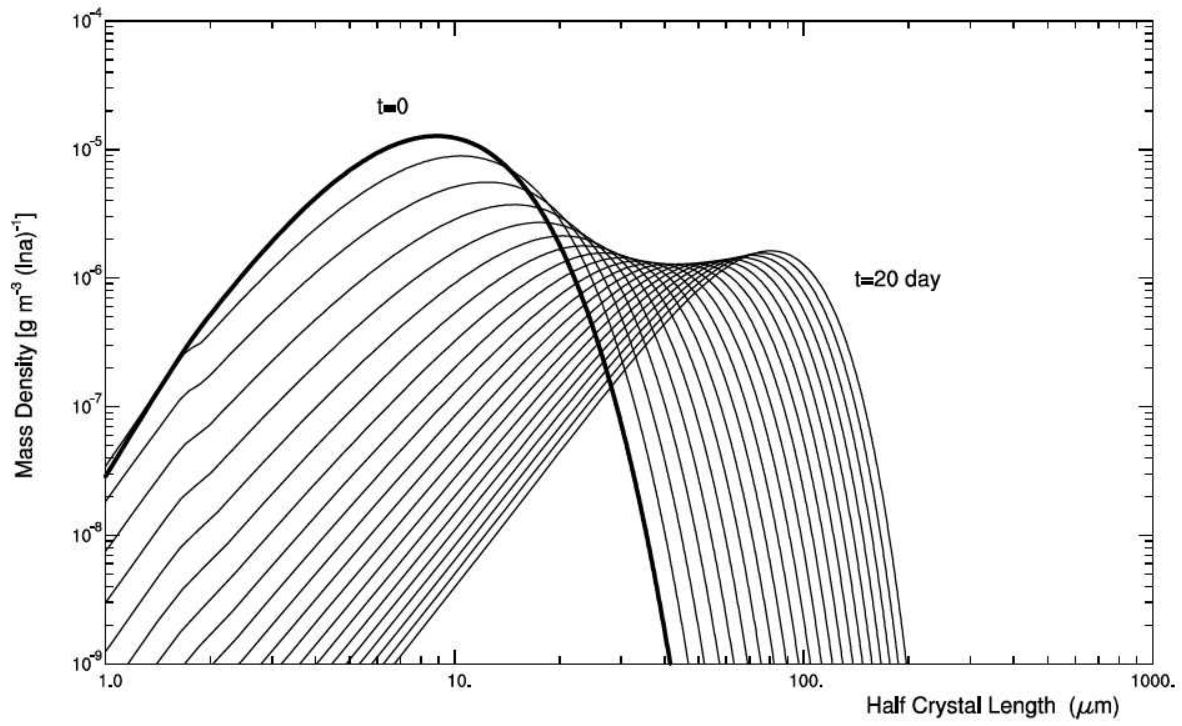

Figure 7 Evolution of the mass density $d M(a) / d \ln a$ versus the half crystal size $a$ in the default experiment where $T=-80^{\circ} \mathrm{C}$ and $p=100 \mathrm{hpa}$. The thick line denotes the initial spectrum, and the time interval between lines is 1 day. 


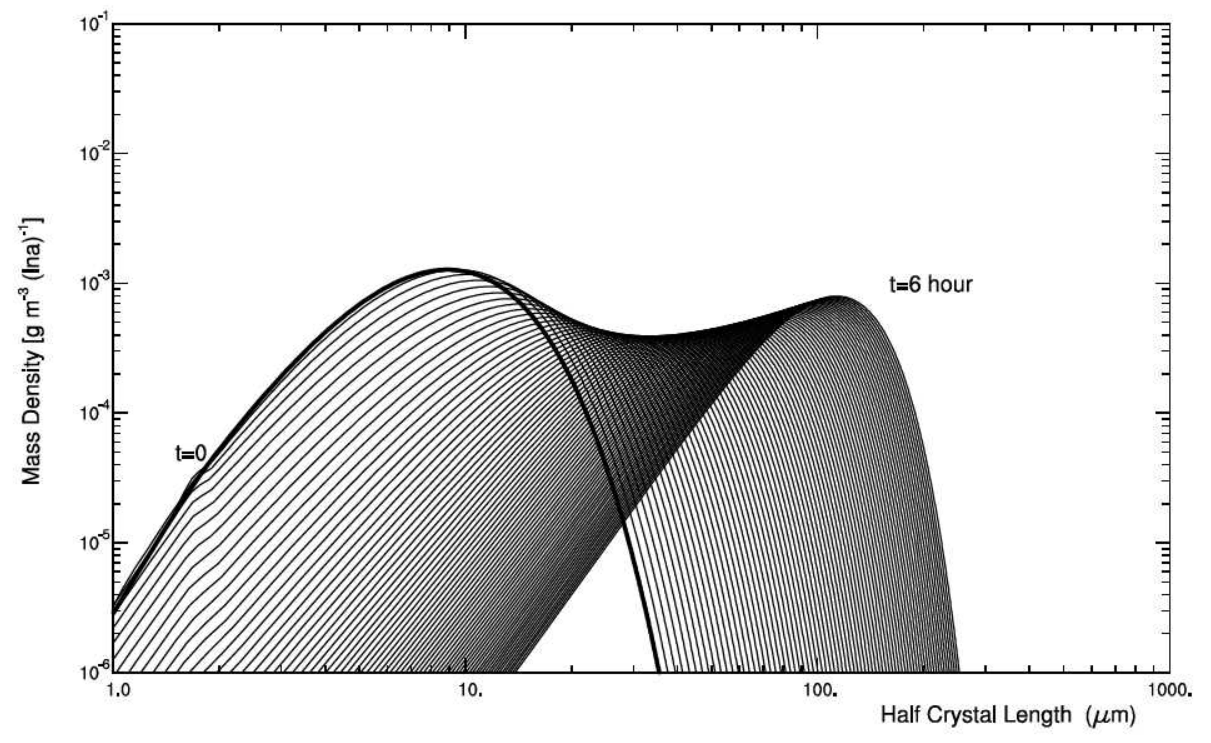

Figure 8 Same as in Fig. 7 except that $T=-42^{\circ} \mathrm{C}, p=250 \mathrm{hpa}$ and the time interval between lines is 5 minutes. 


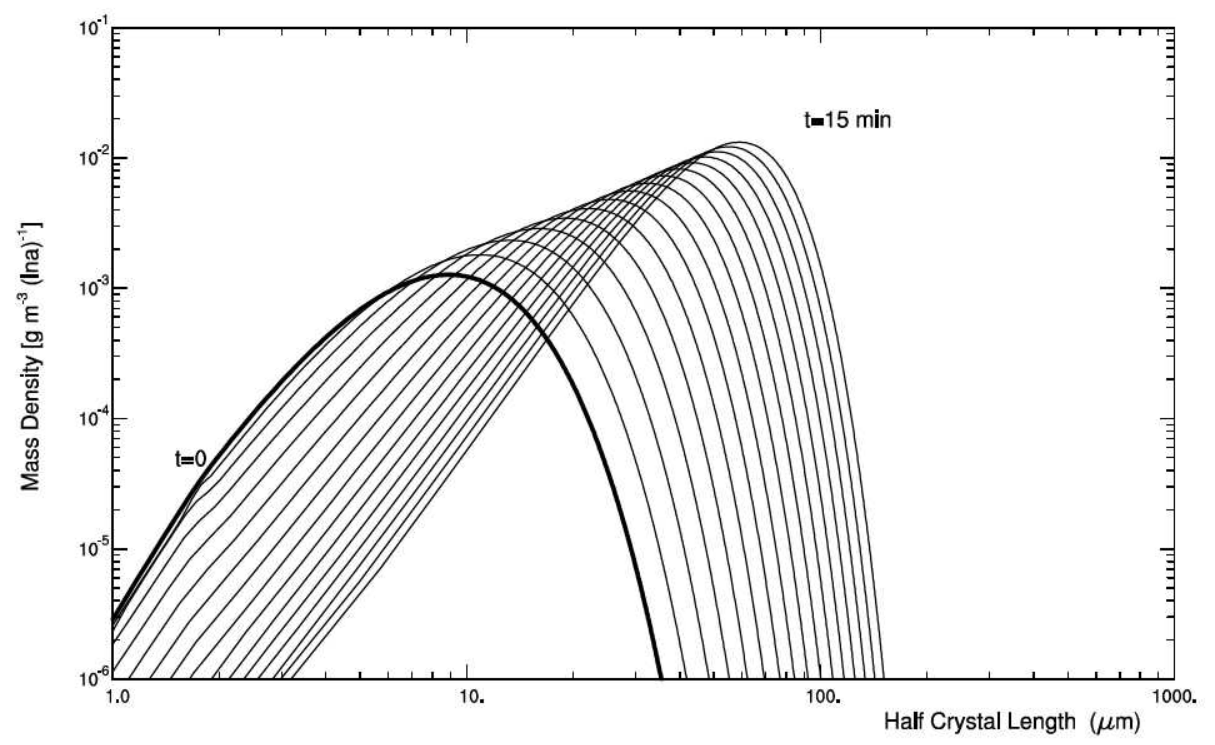

Figure 9 Same as in Fig. 7 except that $T=-6^{\circ} \mathrm{C}, p=500 \mathrm{hpa}$ and the time interval between lines is 1 minute. 


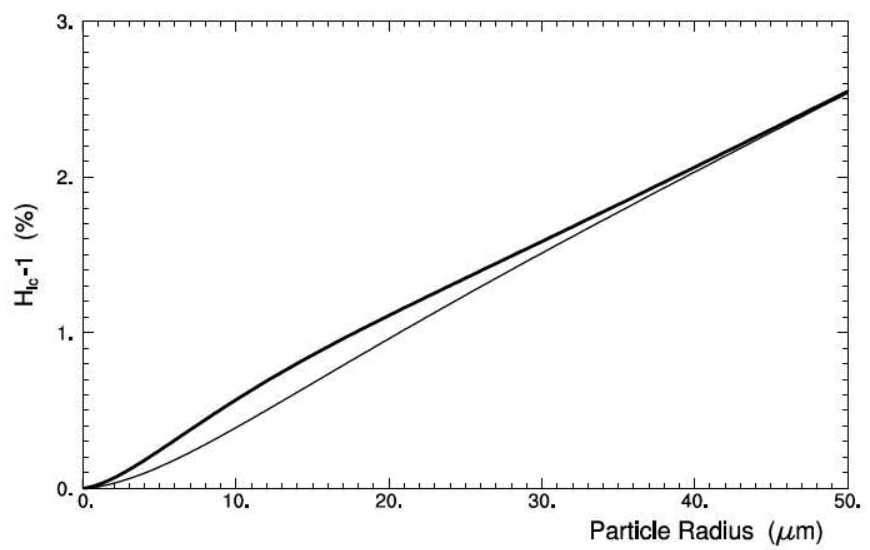

Figure 10 Critical relative humidity $H_{i c}$ as a function of particle radius $r^{*}$, where $\eta=1.8$, $T=-80^{\circ} \mathrm{C}, p=100 \mathrm{hpa}$, and $T_{u s t}=300 \mathrm{~K}$. The thin and thick lines represent $H_{i c}$ when $\varepsilon_{r}$ equals one and is calculated with (5.1), respectively. 


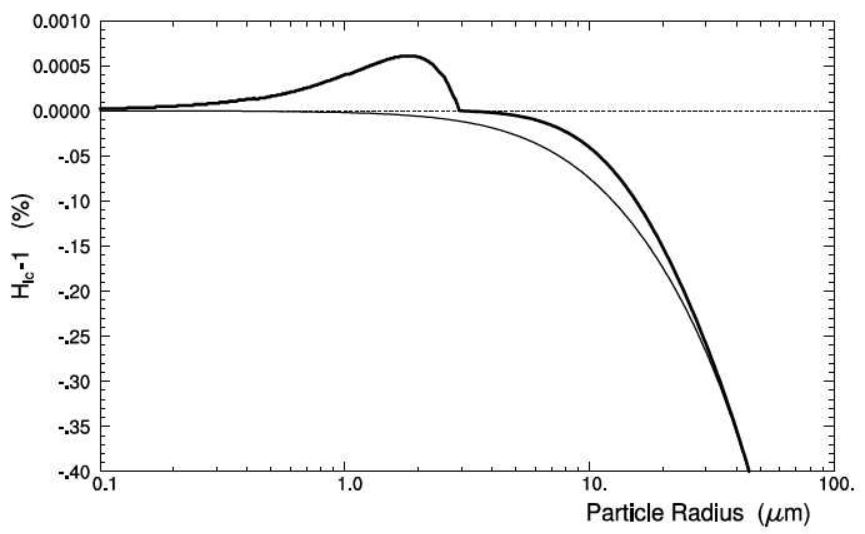

Figure 11 Critical relative humidity $H_{i c}$ as a function of particle radius $r^{*}$, where $\eta=0.9$, $T=-12^{\circ} \mathrm{C}, p=430 \mathrm{hpa}$, and $T_{u s}=300 \mathrm{~K}$. The thin and thick lines represent $H_{i c}$ when $\varepsilon_{r}$ equals one and is calculated with (5.1), respectively. 


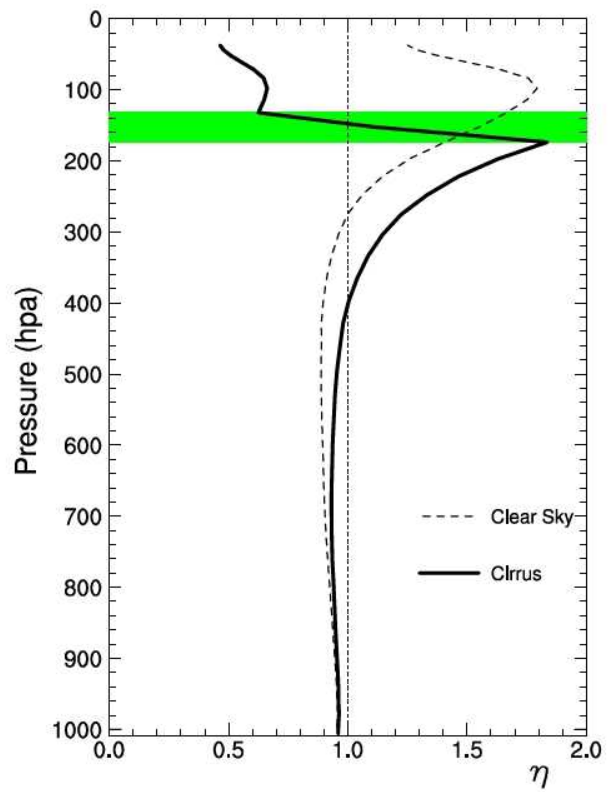

Figure 12 Infrared ratio $\eta$ as a function of pressure for two cases. The thick line represents a case for a cloud (denoted by the shaded area). The dashed line represents a case for a clear sky. 


\title{
The Influence of Radiation on Ice Crystal Spectrum in the Upper Troposphere
}

\author{
Xiping Zeng \\ Goddard Earth Sciences and Technology Center, University of Maryland, Baltimore \\ County, and Laboratory for Atmospheres, NASA Goddard Space Flight Center, \\ Greenbelt, Maryland, USA
}

Published in Quarterly Journal of the Royal Meteorological Society

\section{Popular Summary}

Cirrus clouds impact radiation and therefore the global energy cycle greatly. However, their precipitation mechanism is still unclear, which leads to excessive water in the upper troposphere in current cloud and climate models. This theoretical study is carried out to investigate the effect of radiation on ice crystal spectrum in the upper troposphere. It reveals a new precipitation mechanism in the upper troposphere and the stratosphere, which well explains the subvisual clouds near the tropopause. 